\title{
How to account for the possibility of disguise when assessing signature comparisons
}

\author{
RAYMOND MARQUIS \\ School of Criminal Justice, University of Lausanne, Switzerland \\ TACHA HICKS \\ ORCID: 0000-0002-0114-5258 \\ School of Criminal Justice, University of Lausanne, Switzerland \\ WiLliams MAZZELLA \\ School of Criminal Justice, University of Lausanne, Switzerland
}

\begin{abstract}
Following the recommendations of the ENFSI guideline for evaluative reporting, forensic handwriting examiners should assign a LR to evaluate their results. But when evaluating the results of a signature analysis, how should one account for the possibility of disguise? The present paper explores three possible solutions. The first option could be to assign two LR values, one assuming that the questioned signature was disguised, and the other that it was sincerely written. The authors show that this option is not optimal, since propositions ought to be exhaustive in the context of the case. It is the expert's task to take into account all the relevant information to assess results in a meaningful way. The second option suggests partitioning the proposition of a genuine signature based on the event of disguise and non-disguise. This requires assigning probabilities of disguise and non-disguise and could impact the value of the results. The third option is to
\end{abstract}


consider only the events that have an impact on the case and not formalising those that do not solely for the sake of exhaustiveness. These developments will hopefully help forensic handwriting examiners to cope with the possibility of disguise when assessing the value of signatures in casework.

Keywords: handwriting examination, signature analysis, forensic interpretation, likelihood ratio, Bayesian framework

\section{Introduction}

In 2015, the European Network of Forensic Science Institutes (ENFSI) published a guideline for evaluative reporting in forensic science. ${ }^{1}$ This guideline recommends following the likelihood ratio (LR) approach for assessing the value of forensic results. This method typically should be adopted in cases where an evaluation needs to be carried out by a forensic scientist after having compared a questioned item and reference material from a known source.

More and more laboratories in Europe and abroad are moving towards the LR approach, which is now well documented and developed in many forensic disciplines, such as forensic genetics, micro-traces, fingerprints, firearms, and handwriting analysis. The method provides a standardized basis and a common language between experts, which is in agreement with scientific requirements. The desired properties of forensic scientists' statements, which include balance, logic, robustness, and transparency, can be met by following the principles of forensic interpretation, ${ }^{2}$ also highlighted in the guideline.

The guideline introduces the framework in which the method is applied, exposes principles ${ }^{3}$ and concepts, gives advice on communicating

1 S. Willis et al., ENFSI guideline for evaluative reporting in forensic science, 2015, http://enfsi.eu/wp-content/uploads/2016/09/m1_guideline.pdf.

2 I.W. Evett et al., "The impact of the principles of evidence interpretation on the structure and content of statements", Science and Justice, 40, 2000, pp. 233-239.

3 According to these principles, the assessment is always carried out in a set of circumstances (and relevant information must be taken into account), it is based on two propositions at least (which generally represent the views of the parties at trial), and the forensic scientist evaluates the results (in the light of the propositions) and not the propositions themselves (in the light of the results). 
the value of forensic results. It also provides some examples in various disciplines of forensic science to help readers understand the method. However, the guideline is not designed to help forensic scientists assign a LR given case specifics, nor to assist forensic scientists in eliciting probabilities that take part in their LR. Without appropriate education it is therefore difficult for forensic scientists to apply the approach in practice. This difficulty lies not only in misunderstanding what uncertainty is and how to measure it (i.e. probabilities), but rather in the degree of detail that must be reached in the LR formula development. This is especially true in regard to what factors need to be accounted for and how.

In short, challenges may be faced when turning theory into practice and assigning LRs in casework. This paper deals with one problem commonly encountered by the authors in the area of forensic handwriting examination, more specifically - cases where there is a chance that the true writer has disguised the questioned signature. It may indeed be challenging to know how to properly handle such a possibility (i.e., to logically take into account this event in the assessment of the comparison of signatures). This drawback will be addressed through a fictive case, and solutions will be proposed.

It is worth noting that the present article will not explain how to elicit probabilities that take part in the LR. The values given will serve as a basis for discussion. Interested readers may find relevant information on elicitation of probabilities in the context of signature analysis in Köller et al. ${ }^{4}$ and Marquis et al. ${ }^{5}$

\section{The case}

Let us consider a signature case where a lot of similarities and no differences are observed between the questioned and reference signatures. Let us denote our comparison results by the letter ' $E$ ', for evidence. To evaluate our results, in agreement with the ENFSI guideline, we con-

4 N. Köller et al., Probabilistische Schlussfolgerungen in Schriftgutachten [Probability conclusions in expert opinions in handwriting], München 2004;

5 R. Marquis et al., "What is the error margin of your signature analysis?", Forensic Science International 281, 2017, e1-e8. 
sider two mutually exclusive propositions, exhaustive in the context of the case at hand. ${ }^{6}$

$H_{1}$ - the questioned signature was written by Mr. Jones.

$\mathrm{H}_{2}$ - the questioned signature was simulated by an unknown person.

In its usual simple form, the LR formula can be written as follows: $L R=$ $P\left(E \mid H_{1}, I\right) / P\left(E \mid H_{2}, I\right)$. Let us assume the results are very likely if Mr. Jones wrote the questioned signature, and consider a value close to 1 for the numerator of the LR. At the same time, let us assume the results are very unlikely in the case of a simulation, given the signature's complexity. Let us consider a value of 0,001 for the denominator of the LR. The LR value is therefore $1000(=1 / 0,001)$. Disguise was not considered in this evaluation.

\section{Taking disguise into account}

Let us consider that a new piece of information has been delivered in the case at hand and the expert is now required to take into account the possibility that the questioned signature was disguised. How does one include the possibility of disguise in the assessment of the results? Three options are proposed hereafter.

\section{First option}

The first solution involves assuming that it is not the role of the expert to consider the intentions of the writer. In this view, the results may be assessed separately under two sets of propositions. The first set assumes that the questioned signature, if written by Mr. Jones, has been sincerely written (i.e., without disguising it):

$H_{1 a}$ - the questioned signature was sincerely written by Mr. Jones.

$H_{2 a}$ - the questioned signature was simulated by an unknown writer.

The second set assumes that the questioned signature, if written by Mr. Jones, has been deliberately modified (i.e. disguised).

$H_{1 b}$ - the questioned signature was disguised by Mr. Jones.

$\mathrm{H}_{2 b}$ - the questioned signature was simulated by an unknown writer.

6 A. Biedermann et al., "On the use of the likelihood ratio for forensic evaluation: Response to Fenton et al.”, Science \& Justice 54, 2014, pp. 316-318. 
Two separate LR values would be provided and the responsibility of deciding which one is relevant in the case at hand would be left to the decision maker (e.g. the judge). If this solution was adopted, the meaning of these two values would need to be stated in a clear and transparent way, so that the judge could use them properly. However, one can wonder whether it is relevant to report two different LR values in a case and whether it is realistic to expect a coherent use of the expert's conclusions from the judge, since the task is already not straightforward with a single $L R$ value.

Depending on the case, it may, of course, happen that the two LR values differ significantly. Such a potential difference demonstrates that the possibility of disguise can be a valuable information to take into account. Any information that strongly impacts the LR value should be considered as an important factor that needs to be taken into account when assessing the results in the case. Otherwise, one may consider the situation where the expert may deliver one LR value for each separate important factor, which is definitely not acceptable. It is the role of the expert to consider all the relevant information to express the strength of the results.

\section{Second option}

The second option is to formulate the propositions slightly differently, by partitioning the proposition appearing on the numerator based on disguise. ${ }^{7}$

$H_{1}$ - the questioned signature was written - with or without disguise - by Mr. Jones.

$\mathrm{H}_{2}$ - the questioned signature was simulated by an unknown person.

The formal inclusion of the possibility of disguise in the LR numerator requires one to apply the law of total probability, also called the law of extension of the conversation. ${ }^{8}$ The LR can therefore be written as follows (here we omit the information for ease of notation in the formula and we denote disguise by $D$ and non-disguise by $\bar{D}$ ).

7 N. Köller et al., op. cit.; A. Biedermann, R. Voisard, F. Taroni, "Learning about Bayesian networks for forensic interpretation: an example based on 'the problem of multiple propositions"', Science \& Justice 52, 2012, pp. 191-198.

8 C. Aitken, F. Taroni, Statistics and the evaluation of evidence for forensic scientists, Chichester 2004. 


$$
L R=\frac{P\left(E \mid H_{1}, D\right) \times P\left(D \mid H_{1}\right)+P\left(E \mid H_{1}, \bar{D}\right) \times P\left(\bar{D} \mid H_{1}\right)}{L R=P\left(E \mid H_{2}\right)}
$$

The probability of the results if $H_{l}$ holds is now the sum of two terms: (1) the probability of the evidence conditioned on disguise multiplied by the probability of disguise, and (2) the probability of the evidence conditioned on non-disguise multiplied by the probability of non-disguise. These conditioned probabilities of the results must thus be respectively multiplied by probabilities $P\left(D \mid H_{1}\right)$ and $P\left(\bar{D} \mid H_{1}\right)$ that Mr. Jones did - or did not - disguise his signature. We know that these two probabilities add to 1 , because the events are mutually exclusive and exhaustive. The forensic handwriting examiner, who may have no information about such probabilities, could suppose that disguise is just as likely as non-disguise, and assign values of 0,5 to each of the probabilities. In the case at hand, since the findings are not expected in the case of disguise, a value close to zero is assigned to the probability of the results under disguise $P\left(E \mid H_{1}, \bar{D}\right)$. As the findings are very likely if Mr. Jones wrote the questioned signature without disguising it, a value close to 1 is assigned to the probability $P\left(E \mid H_{1}, \bar{D}\right)$. The LR finally approximately becomes:

$$
L R=\frac{0 \times 0,5+1 \times 0,5}{\mathrm{LR}=0,001=500}=500
$$

Remember that if disguise was not considered at all, a value of 1 would have been assigned to the numerator of the LR. In other words, by partitioning the proposition of the numerator based on disguise, a level of uncertainty that is difficult to handle quantitatively has been introduced, and a decrease of the LR value is observed (from 1000 to 500) due to the probabilities of the considered sub-propositions. Therefore, adding uncertainty about the writing conditions of the signature has an impact on the LR value, though it can be argued this effect is only moderate in this case since the LR value remains within the same order of magnitude. It is worth mentioning that the same kind of inconvenience would arise if uncertainty were to be formalised regarding the type of simulation (free hand, tracing, and so on) and taken into account in the denominator of the LR. 
It is also worth mentioning that the features of the questioned signature (i.e. without comparing it with the reference signatures) may inform the forensic handwriting examiner about the probability of disguise. An example of this would be the presence of hesitation or retouching. ${ }^{9}$ Taken into account formally, this element would make the LR formula more complex - however, this will not be developed here.

\section{Third option}

Finally, it may be assumed that there is no need to formalise uncertainty about the writing conditions in the LR. Unless this was explicitly requested by the mandating authority or known to be important for the decision making, no sub-propositions would be specified. But of course, the possibility of disguise may be taken into account to assign a value to the LR numerator, exactly in the same way other information would be taken into account, such as the writer's health status, whether they were sitting at a table, whether they take abusive substances, etc. All of this information is necessarily taken into consideration during the evaluation process, though not necessarily made explicit in the propositions. However, one should bear in mind that assumptions made in the evaluation stage should be disclosed in the statement. The recipient of information should know, for example, whether disguise was taken into account or excluded based on the case circumstances. Following this third solution, the propositions are left unchanged compared to the initial set (i.e. "the questioned signature was written by Mr. Jones" vs "the questioned signature was simulated by an unknown person"). On the one hand, this could mean putting a weight of one or zero on disguise, whether disguise is respectively taken as true or not. In this case, the assumptions regarding (non-)disguise must be disclosed in the expert's statement. On the other hand, this could mean that the probability of the results is globally assessed given both disguise and non-disguise, but without formally extending the conversation. The authors argue that for transparency and robustness, if the probability of disguise is not close to zero or one, then the second approach proposed in this paper is recommended.

9 R.A. Huber, A.M. Headrick, Handwriting identification: Facts and fundamentals, Boca Raton, FL 1999. 


\section{Conclusion}

In the future, forensic laboratories may be requested to deliver the strength of forensic results in the form of an LR in their evaluative statements. This is supported by a recently published guideline on the matter by the ENFSI. While the LR approach is theoretically appealing, its application in practice is challenging, as any scientifically sound evaluation would be. One major issue was addressed in the present paper to help handwriting examiners who may face a problem typical for their discipline: how to incorporate the possibility that a questioned signature was disguised into the LR. The first option proposed is to deliver two LR values, one assuming that the questioned signature was disguised, and the other assuming it was sincerely written. The authors argue this option is problematic because disguise may significantly affect the LR, and it is especially the expert's task to account for all the relevant information when assessing the value of the results. The delivery of two LR values may furthermore be embarrassing for the recipient of information. A second option would be to partition the proposition of a genuine signature (on the numerator of the LR) based on the event of disguise vs. nondisguise. This procedure requires the assignment of probabilities of disguise and non-disguise, which may be logical, but also laborious. This procedure will impact one's LR - although in a limited way - compared to the situation where disguise is not made explicit in the propositions. In the third option, in cases where disguise can be either admitted or excluded (based on case circumstances), there is no need to explicitly mention disguise in the propositions. However, the assumption about (non-)disguise should be disclosed in the expert's statement. These developments will hopefully help forensic handwriting examiners to cope with the problem of taking disguise into account in their evaluation. While the task may be challenging, we encourage forensic handwriting examiners to adopt the logical approach recommended by the ENFSI, which will certainly contribute to harmonising and structuring the assessment of findings to strengthen conformity to scientific requirements. 


\section{References}

Aitken C., Taroni F., Statistics and the evaluation of evidence for forensic scientists, Chichester 2004.

Biedermann A. et al., "On the use of the likelihood ratio for forensic evaluation: Response to Fenton et al.", Science \& Justice 54, 2014, pp. 316-318.

Biedermann A., Voisard R., Taroni F., "Learning about Bayesian networks for forensic interpretation: An example based on 'the problem of multiple propositions"”, Science \& Justice 52, 2012, pp. 191-198.

Evett I.W. et al., "The impact of the principles of evidence interpretation on the structure and content of statements", Science and Justice, 40, 2000, pp. 233-239.

Huber R.A., Headrick A.M. Handwriting identification: Facts and fundamentals, Boca Raton, FL 1999.

Köller N. et al., Probabilistische Schlussfolgerungen in Schriftgutachten [Probability conclusions in expert opinions in handwriting], München 2004.

Marquis R. et al., "What is the error margin of your signature analysis?", Forensic Science International 281, 2017, e1-e8.

Willis S. et al., ENFSI guideline for evaluative reporting in forensic science, 2015, http:// enfsi.eu/wp-content/uploads/2016/09/m1_guideline.pdf. 